\title{
Real Interference Alignment and Degrees of Freedom Region of Wireless X Networks
}

\author{
Zhengdao Wang \\ Department of Electrical and Computer Engineering, Iowa State University, Ames, IA 50011 USA \\ e-mail: zhengdao@iastate.edu
}

\begin{abstract}
We consider a single hop wireless $\mathbf{X}$ network with $K$ transmitters and $J$ receivers, all with single antenna. Each transmitter conveys for each receiver an independent message. The channel is assumed to have constant coefficients. We develop interference alignment scheme for this setup and derived several achievable degrees of freedom regions. We show that in some cases, the derived region meets a previous outer bound and are hence the DoF region. For our achievability schemes, we divide each message into streams and use real interference alignment on the streams. Several previous results on the DoF region and total DoF for various special cases can be recovered from our result.
\end{abstract}

Index Terms - real interference alignment, degrees of freedom region, wireless $X$ network, stream alignment

\section{INTRODUCTION}

The wireless $\mathrm{X}$ network [1], models a single-hop wireless network such that each transmitter conveys an independent message for each receiver. All transmitter and all receivers have single antenna. Multiple antenna extensions have been considered [2]. The $\mathrm{X}$ network model includes the broadcast channels, multiple access channels, and the interference channels as special cases. It is therefore useful to quantify the capacity limits of $X$ networks. However, this is a difficult problem because even the capacity region for the broadcast channel, which is a special case of the $\mathrm{X}$ network, has not been characterized in full generality (e.g., discrete memoryless broadcast channel).

Simple single-letter type characterizations of capacity regions for many of other multi-user information-theoretic problems have also eluded us. A recent line of attack focuses on Gaussian networks in the asymptotic regime where the signal to noise ratio (SNR) goes to infinity. The communication rates are normalized by $\log (\mathrm{SNR})$ to yield a quantity defined as the degrees of freedom (DoF), or multiplexing gain [3]. The shape of the capacity region normalized by $\log (\mathrm{SNR})$ as SNR goes to infinity is defined as the DoF region, e.g., [2]. The total DoF and in some cases the DoF region for several channels have been characterized recently. One important technique for proving the achievability results is the interference alignment, which seeks to align the dimensions of interference signals so that more dimensions are available in the subspace unaffected by interference.

There are several interference alignment techniques, among which are the vector interference alignment based on beamforming and zero-forcing, e.g., [4], [5], and the real interference alignment [6]-[9]. There seems to be intimate connections between the two methods.
For the DoF problem of wireless $\mathrm{X}$ network, several results are available. An outer bound for multiple-input multipleoutput (MIMO) X network has been derived in [1], which also developed schemes for achieving the maximum total DoF for single antenna $\mathrm{X}$ network. For constant single-antenna channels, a real interference alignment scheme has been used in [9] to establish the maximum total DoF. For MIMO X networks, outer bounds and achievability schemes have been developed in [2] for the $2 \times 2$ MIMO $\mathrm{X}$ network. The DoF region for an $M \times 2 \mathrm{X}$ network with $N_{1}$ and $N_{2}$ antennas at the two receivers is available as a special case of the result in [10]. Antenna splitting argument has been used in [1] to establish a lower bound on the total DoF of MIMO X network.

In this paper, we focus on the single-antenna wireless $\mathrm{X}$ networks, and derive several achievability schemes based on real interference alignment. The achieved DoF regions are shown to be tight when the number of receivers is two. Several previous results (or their constant channel counter parts) can be recovered as special cases.

\section{SYSTEM MODEL}

Notation: Throughout the paper, $J$ and $K$ will be integers and $\mathcal{J}=\{1, \ldots, J\}, \mathcal{K}=\{1, \ldots, K\}$. We use $k, \tilde{k}, \hat{k}$ as transmitter indices, and $j, \tilde{j}, \hat{j}$ as receiver indices. The set of integers and real numbers are denoted as $\mathbb{Z}$ and $\mathbb{R}$, respectively. We use $\left[d_{j, k}\right]$ to denote a matrix with element $d_{j, k}$ in the $(j, k)$ th position, and use $\left[d_{j, k}\right]_{j=1, k=1}^{J, K}$ to make the size of the matrix explicit. Letter $l$ will be reserved for the index of streams (parts of a message). Throughout the paper, a.e. means almost everywhere in the Lebesgue sense for the channel matrix.

Consider a single-antenna wireless $\mathrm{X}$ network with $K$ transmitters and $J$ receivers. For each pair $(j, k) \in \mathcal{J} \times \mathcal{K}$, transmitter $k$ conveys a message $m_{j, k}$ for receiver $j$. The channel from transmitter $k$ to receiver $j$ is denoted as $h_{j, k}$. The whole set of channel coefficients is denoted as a matrix

$$
\mathbf{H}:=\left[h_{j, k}\right]_{j=1, k=1}^{J, K} .
$$

All the quantities are real in this paper. So $\mathbf{H} \in \mathbb{R}^{J \times K}$. The channel is assumed constant (non-fading) throughout the whole transmission. Each transmitter $k$ transmits a symbol $x_{k, t}$ in time slot $t \in \mathbb{Z}$. Each transmitter has an average power constraint $P$ so that for any transmission that spans $N \in \mathbb{Z}$ symbols, the transmitted symbols satisfy

$$
\sum_{t=1}^{N} \frac{1}{N}\left|x_{k, t}\right|^{2} \leq P, \quad \forall 1 \leq k \leq K .
$$


The received signal at receiver $j$ at time $t$ can be written as

$$
y_{j, t}=\sum_{k \in \mathcal{K}} h_{j, k} x_{k, t}+\nu_{j, t}, \quad \forall j \in \mathcal{J}
$$

where $\left\{\nu_{j, t} \mid j \in \mathcal{J}\right\}$ is the set of additive noises, assumed to be independent and identically distributed according to zero mean Gaussian distribution with unit variance. So $P$ is the per-message SNR.

A code of length $N$ and message sizes $\left[M_{j, k}\right]$ consists of

1) the encoders $\left\{f_{k} \mid k \in \mathcal{K}\right\}$, where $f_{k}$ is a mapping from the set of messages to be conveyed by transmitter $k,\left[1, M_{1, k}\right] \times, \ldots, \times\left[1, M_{J, k}\right]$, to the set of transmitted symbols (codewords) in $\mathbb{R}^{N}$. All codewords satisfy the power constraint.

2) the decoders $\left\{g_{j, k} \mid j \in \mathcal{J}, k \in \mathcal{K}\right\}$, where $g_{j, k}$ is a mapping from the set $\mathbb{R}^{N}$ of received symbols at receiver $j$ to the set of messages $\left[1, M_{j, k}\right]$ intended for receiver $j$ from transmitter $k$.

The rate of message $m_{j, k}$ is defined to be

$$
R_{j, k}=\frac{1}{N} \log _{2}\left(M_{j, k}\right) .
$$

Let $\left[W_{j, k}\right]$ denote a set of messages such that $W_{j, k}$ is independently and uniformly chosen from $\left[1, M_{j, k}\right]$. The probability of error $P_{e}$ of the code is defined as

$$
\begin{array}{r}
\operatorname{Pr}\left[g_{j, k}\left(\sum_{k \in \mathcal{K}} h_{j, k} f_{k}\left(\left[W_{j, k}\right]_{j=1}^{J}\right)+\left[v_{j, t}\right]_{t=1}^{N}\right) \neq W_{j, k}\right. \\
\text { for some }(j, k) \in \mathcal{J} \times \mathcal{K}] .
\end{array}
$$

The code we have thus defined will be denoted as a

$$
\left(P, N,\left[M_{j, k}\right],\left[f_{k}\right],\left[g_{j, k}\right]\right)
$$

code.

The degree of freedom (DoF) region for the system is the closure of a set of points $\left[d_{j, k}\right] \in \mathbb{R}^{J \times K}$ such that for any $\epsilon>0$, there is a sequence of codes $\left(P^{(i)}, N^{(i)},\left[M_{j, k}^{(i)}\right],\left[f_{k}^{(i)}\right],\left[g_{j, k}^{(i)}\right]\right)$ indexed by $i$ such that as $i \rightarrow \infty$, the power $P^{(i)} \rightarrow \infty$, and

$$
\lim _{i \rightarrow \infty} \frac{R_{j, k}^{(i)}}{0.5 \log P^{(i)}}=d_{j, k}, \quad \forall j \in \mathcal{J}, \forall k \in \mathcal{K},
$$

and such that for all $i$, the probability of error is less than $\epsilon$.

\section{Achievable Degrees of Freedom Region}

\section{A. Statement of result}

Theorem 1 (An achievable DoF region). For a $K$-transmitter $J$-receiver constant-coefficient single-antenna wireless $X$ network $\mathbf{H} \in \mathbb{R}^{J \times K}$, the DoF region $\mathcal{D}$ satisfies $\mathcal{D} \supset \mathcal{D}^{\text {(in) }}$ a.e., where $\mathcal{D}^{(i n)}$ is a set of matrices $\left[d_{j, k}\right]_{j=1, k=1}^{J, K}$ such that

1) all entries of it are non-negative;

2) $\forall 1 \leq j \leq J$, the following inequality holds:

$$
\sum_{\tilde{k}=1}^{K} d_{j, \tilde{k}}+\sum_{\tilde{j} \in \mathcal{J}, \tilde{j} \neq j} \max _{k} d_{\tilde{j}, k} \leq 1 .
$$

\section{B. Main ideas}

Our achievability proof uses the following ideas:

1) We use real interference alignment, a technique that has been initiated in [6], and further extended for the interference problems in [7]-[9].

2) We split each message into streams, where all streams have the same have the same DoF. This allows us to design achievability schemes for unequal DoFs. This idea has been used in e.g., [11].

3) The interference alignment at the receivers is streambased. Several streams from different transmitters are aligned. Streams from the same transmitter are never aligned. Otherwise decodability of the aligned messages at other receivers will be compromised.

4) We use a construction that involves "dimension padding" to guarantee that all streams have the same DoF.

\section{The proof}

We prove that for any $\left[d_{j, k}\right] \in \mathcal{D}^{(\text {in) }},\left[d_{j, k}\right]$ is achievable. We assume that all the elements of $\left[d_{j, k}\right]$ are rational numbers. Otherwise, if some elements are irrational, the proof here can be used to establish achievability of a point that is arbitrarily close to $\left[d_{j, k}\right]$.

Under the rational assumption, we can find an integer $\kappa$ such that for all $j \in \mathcal{J}$ and all $k \in \mathcal{K}, \bar{d}_{j, k}:=\kappa d_{j, k}$ is a non-negative integer.

ENCODING: For each $(j, k) \in \mathcal{J} \times \mathcal{K}$, the message $m_{j, k}$ is divided into $\bar{d}_{j, k}$ parts as $\left\{m_{j, k, l}, l=1, \ldots, \bar{d}_{j, k}\right\}$. Each part is called a stream. The signal emitted by transmitter $k$ is in the following form

$$
x_{k}=\sum_{j \in \mathcal{J}} x_{j, k}=\sum_{j \in \mathcal{J}} \sum_{l=1}^{\bar{d}_{j, k}} \alpha_{j, k, l} x_{j, k, l}
$$

where $x_{j, k, l}$ carries the symbols of stream $l$ of the message from transmitter $k$ to receiver $j$, and $\left\{\alpha_{j, k, l}\right\}$ are design parameters that can be chosen randomly, independently, and uniformly from the interval $\left[\frac{1}{2}, 1\right]$. The symbol $x_{j, k, l}$ is generated using elements (called directions [9]) specified in a set $\mathcal{T}_{j, k, l}$ (to be specified later) as follows:

$$
x_{j, k, l}=\sum_{\delta_{b} \in \mathcal{T}_{j, k, l}} \delta_{b} u_{j, k, l, b}
$$

where $u_{j, k, l, b} \in\{\lambda q \mid q \in \mathbb{Z},-Q \leq q \leq Q\}$, and $Q$ and $\lambda$ are parameters to be specified appropriately later to satisfy the rate and power requirements. In the summation in (9), we have assumed that the directions in $\mathcal{T}_{j, k, l}$ have been indexed from 1 , and $b$ is the index of the direction of $\delta_{b}$. The exact indexing scheme is of no importance.

Stream Alignment: Consider an arbitrary receiver $\hat{j}$. The signal dimensions situation is shown in Fig. 1. The useful signals have DoF $\sum_{k \in \mathcal{K}} \bar{d}_{\hat{j}, k}$. The interferences coming from different transmitters are shown on the right. The streams intended for the same receiver $j \neq \hat{j}$ are aligned together at receiver $\hat{j}$.

DIMENSION PADDING: To facilitate the construction of the transmission directions, we introduce an idea that we term 


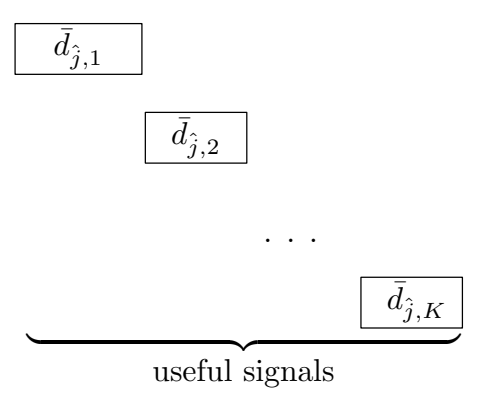

useful signals aligned stream

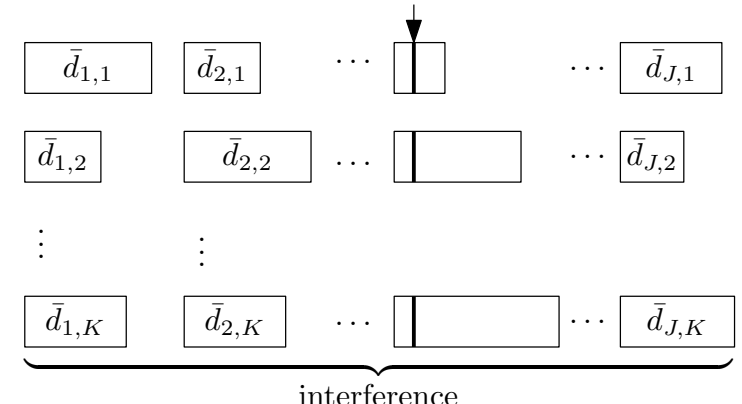

Fig. 1. Interference alignment at receiver $\hat{j}$

dimension padding. Specifically, we notice that in the interference part in Fig. 1, the messages intended for the same receiver $j \neq \hat{j}$ in general do not have the same number of streams. To make sure that such disparity does not lead to difference in the achieved DoF for these messages, we introduce some fictitious streams so that with these additional streams the constructed transmission symbols for all actual streams use the same number of directions. These fictitious streams only aid in the construction of the transmission directions. No symbols are transmitted for these streams, otherwise the useful signal space dimension will become smaller (the interference space dimension remains unchanged though).

More specifically, we assume all messages $\left\{m_{j, k} \mid k \in \mathcal{K}\right\}$ intended for receiver $j$ has the same number $s_{j}$ of streams, where

$$
s_{j}=\max _{k} \bar{d}_{j, k}
$$

For transmitter $k$, the first $\bar{d}_{j, k}$ of these $s_{j}$ streams are actual transmitted streams. The remaining ones (if any) are virtual streams, whose transmitted symbols are all set to zero [c.f. (9)]:

$$
u_{j, k, l, b}=0, \quad \forall l \in\left[\bar{d}_{j, k}+1, s_{j}\right] .
$$

We assume that $\alpha_{j, k, l}$ is assigned for a virtual stream in the same way as for an actual stream.

Transmit DiRECTIONS: We design the directions $\mathcal{T}_{j, k, l}$ used by stream $m_{j, k, l}$ to contain and only contain directions of the following form:

$$
T=\prod_{\hat{j} \in \mathcal{J}, \hat{j} \neq j} \prod_{\hat{k} \in \mathcal{K}}\left(h_{\hat{j}, \hat{k}} \alpha_{j, \hat{k}, l}\right)^{p_{\hat{j}, \hat{k}, j, k, l}}
$$

where

$$
0 \leq p_{\hat{j}, \hat{k}, j, k, l} \leq n-1,
$$

$\forall \hat{j} \in \mathcal{J}, \hat{j} \neq j, \forall \hat{k} \in \mathcal{K}$. It can be seen that there are totally $n^{K(J-1)}$ directions in $\mathcal{T}_{j, k, l}$ for all $(j, k, l)$. The reason for doing dimension padding can be seen more clearly now as it leads to the same number of directions to be used by all streams. This will guarantee that each stream corresponds to the same DoF in the final result.

ALIGNMENT VERIFICATION: The proposed design above guarantees that the interferences created by messages intended for the same receiver are aligned as desired at all receivers. To see this, define $\mathcal{T}_{\hat{j}, j, l}$ to contain directions described by (12) but with

$$
0 \leq p_{\hat{j}, \hat{k}, j, k, l} \leq n,
$$

for all $(\hat{j}, \hat{k}, j, k, i)$. According to (9), a symbol from stream $(j, k, l)$ is transmitted in a direction of the form $\alpha_{j, k, l} T$ where $T$ is as in (12). This symbol will arrive at receiver $\hat{j}$ in the direction of $\left(h_{\hat{j}, k} \alpha_{j, k, l}\right) T$, which is in $\mathcal{T}_{\hat{j}, j, l}$ because the power for $\left(h_{\hat{j}, k} \alpha_{j, k, l}\right)$ will be simply increased by one after the symbol goes through the channel. Note that not all directions in $\mathcal{T}_{\hat{j}, j, l}$ will be occupied by interference so the effective number of interference dimensions is smaller than the number of elements in $\mathcal{T}_{\hat{j}, j, l}$. However, this does not affect the calculation of the achievable DoF.

DECODABILITY: The useful signals at receiver $\hat{j}$ will be generated by directions in $\mathcal{T}_{\hat{j}}^{\prime}$, where

$$
\mathcal{T}_{\hat{j}}^{\prime}=\bigcup_{k \in \mathcal{K}}\left(h_{\hat{j}, k} \alpha_{\hat{j}, k, l}\right) \mathcal{T}_{\hat{j}, k, l}
$$

Since none of the $\mathcal{T}_{\hat{j}, k, l}$ contains a generator $\left(h_{\hat{j}, k} \alpha_{\hat{j}, k, l}\right)$ [recall the condition $\hat{j} \neq j$ in [12]], and for different $k$, $\left(h_{\hat{j}, k} \alpha_{\hat{j}, k, l}\right)$ are different, we conclude that $\mathcal{T}_{\hat{j}}^{\prime}$ is rationally independent of $\bigcup_{j, l} \mathcal{T}_{\hat{j}, j, l}$. Therefore, all the useful signals are decodable in the noiseless case a.e..

The total rational dimensions $D_{\hat{j}}$ of both the useful signals and the interference at any receiver $\hat{j}$ is

$$
D_{\hat{j}} \leq \sum_{\tilde{k}=1}^{K} \bar{d}_{\hat{j}, \tilde{k}} n^{K(J-1)}+\sum_{j \in \mathcal{J}, j \neq \hat{j}} \max _{k} \bar{d}_{j, k}(n+1)^{K(J-1)} .
$$

We define

$$
S=\max _{\hat{j} \in \mathcal{J}}\left(\sum_{\tilde{k}=1}^{K} \bar{d}_{\hat{j}, \tilde{k}}+\sum_{j \in \mathcal{J}, j \neq \hat{j}} \max _{k} \bar{d}_{j, k}\right),
$$

which is an upper bound on the total number of useful signal streams and interference streams (multiple aligned streams are counted as one), maximized over all receivers. For any DoF point in $\mathcal{D}^{(\text {in) }}$ that satisfies (7), we have $S \leq \kappa$. As a result, we have

$$
D_{\hat{j}} \leq S(n+1)^{K(J-1)} \leq \kappa(n+1)^{K(J-1)}
$$


With reference to the constellation symbols in (9), if we choose

$$
\lambda=P^{\frac{1}{2}} / Q
$$

then we can guarantee that the power constraint is satisfied. In addition, if for any $\epsilon \in(0,1)$ we choose as in e.g., [9],

$$
Q=P^{\frac{1-\epsilon}{2(m+\epsilon)}},
$$

where $m$ is an integer, then we can guarantee that the DoF per stream is $\frac{1-\epsilon}{m+\epsilon}$. Choosing $m=\kappa(n+1)^{K(J-1)}$, the hard decoding error probability for the constellation symbols decreases to zero as $P \rightarrow \infty$ due to the Khintchine-Groshev type Theorems, see the discussion in e.g., [8], [9], and the DoF of the message $m_{j, k}$ can be arbitrarily close to

$$
\lim _{n \rightarrow \infty} \frac{\bar{d}_{j, k} n^{K(J-1)}}{\kappa(n+1)^{K(J-1)}}=\frac{\bar{d}_{j, k}}{\kappa}=d_{j, k},
$$

for all $j \in \mathcal{J}$ and $k \in \mathcal{K}$. This completes the proof.

\section{EXTENSIONS}

The alignment scheme presented in Sec. IIII is only one possible alignment schemes within the class of real alignment. We have aligned the messages intended for the same receiver. However this is not always optimal and not necessary either. We propose some extensions of the alignment scheme that can yield potentially larger achievable DoF regions.

\section{A. Permuted alignment}

To see the insufficiency of the alignment scheme in Sec. IIII consider a $3 \times 3 \mathrm{X}$ network. If we set all messages $m_{j, k}$ to have rate zero whenever $j \neq k$, then it becomes a 3 -user interference channel. It is known [5] that per user DoF $1 / 2$ is achievable. Therefore, the following DoF point is within the DoF region of the $3 \times 3 \mathrm{X}$ network:

$$
\left[d_{j, k}\right]^{T}=\left[\begin{array}{ccc}
\frac{1}{2} & 0 & 0 \\
0 & \frac{1}{2} & 0 \\
0 & 0 & \frac{1}{2}
\end{array}\right] .
$$

However, it can be seen that this point cannot be achieved using the scheme in Sec. IIII To achieve this point, we can arrange the individual DoFs in each row so that it looks as follows (c.f. Fig. 11):

$$
\left[\begin{array}{lll}
d_{1,1} & d_{2,1} & d_{3,1} \\
d_{2,2} & d_{1,2} & d_{3,2} \\
d_{3,3} & d_{1,3} & d_{3,3}
\end{array}\right]=\left[\begin{array}{ccc}
\frac{1}{2} & 0 & 0 \\
\frac{1}{2} & 0 & 0 \\
\frac{1}{2} & 0 & 0
\end{array}\right] .
$$

Note the matrix has been shown in its transposed form to agree with the Fig. 10 The permutations applied to different rows can be different. To see that this point is achievable, we can check e.g., the situation at receiver 1 as depicted (for illustration only) in the following

$$
\left[\begin{array}{ccc}
\frac{1}{2} & - & - \\
- & 0 & - \\
- & - & 0
\end{array}\right] \mid\left[\begin{array}{ccc}
- & 0 & 0 \\
\frac{1}{2} & - & 0 \\
\frac{1}{2} & - & 0
\end{array}\right]
$$

where the left part is the signal dimensions, and the right part is for the interference dimensions. The minus signs are a place holder that means "no signal". The dimensions on the left
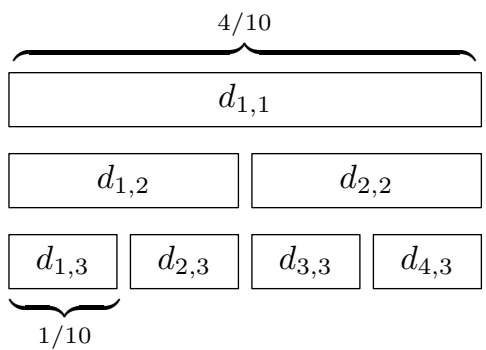

Fig. 2. Staggered alignment

$\left(\frac{1}{2}, 0,0\right)$ are $\left(d_{1,1}, d_{1,2}, d_{1,3}\right)$, the DoF's that receiver 1 needs. These entries have been removed from the right part (replaced with minus signs). Counting the total dimensions by taking the maximum of all the DoF on each column, treating minus as 0 , we have

$$
\frac{1}{2}+0+0+\frac{1}{2}+0+0=1,
$$

which is acceptable. Similar verification can be performed for receiver 2 and 3 as well. As a result, the point as in (21) is achievable. Formally, we state without proof the following.

Theorem 2 (Permuted Alignment). For a $K$-transmitter $J$ receiver constant-coefficient single-antenna wireless $X$ network $\mathbf{H} \in \mathbb{R}^{J \times K}$, the DoF region $\mathcal{D}$ satisfies $\mathcal{D} \supset \mathcal{D}_{0}^{(i n)}$ a.e., where $\mathcal{D}_{0}^{(i n)}$ is a set of matrices $\left[d_{j, k}\right]_{j=1, k=1}^{J, K}$ such that

1) All entries of it are non-negative;

2) There exists $K$ permutations of $J$ objects $\left\{\sigma_{k}(\cdot) \mid k \in \mathcal{K}\right\}$ such that $\forall 1 \leq \hat{j} \leq J$, the following inequality holds:

$$
\sum_{\tilde{k}=1}^{K} d_{\hat{j}, \tilde{k}}+\sum_{\tilde{j} \in \mathcal{J}} \max \mathcal{I}_{\tilde{j}} \leq 1,
$$

where $\mathcal{I}_{\tilde{j}}:=\left\{d_{j, k} \mid k \in \mathcal{K}, j \in \mathcal{J}, j \neq \hat{j}, \sigma_{k}(j)=\tilde{j}\right\}$.

It should be obvious that if we choose the permutations to be the identity mapping (no permutation), then the result in Sec. 1 is recovered. For the purpose of comparison, we will term the alignment scheme in Sec. IIII the natural alignment.

\section{B. Staggered alignment}

In both the natural alignment and the permuted alignment, any message from any single transmitter is aligned with one and only one message from another transmitter. However, this can be generalized. It is possible to align two users' messages so that one message from the first user is aligned with multiple messages from the other user.

Staggered alignment can achieve DoF point that are not achievable using the natural or permuted alignments. Consider a $3 \times 4 \mathrm{X}$ network. The point $\left[d_{j, k}\right]$ as follows

$$
\left[d_{j, k}\right]^{T}=\frac{1}{10}\left[\begin{array}{cccc}
4 & 0 & 0 & 0 \\
2 & 2 & 0 & 0 \\
1 & 1 & 1 & 1
\end{array}\right]
$$

is in the DoF region. This can be established using a staggered alignment scheme as shown in Fig. 2. Using permuted alignment without message staggering, a DoF point that is proportional to the matrix in (26) will have a coefficient $1 / 11$ instead of $1 / 10$ in front. 


\section{DISCUSSION}

\section{A. Some special cases}

An outer bound for the wireless $\mathrm{X}$ channel has been derived in [2]. It states that $\forall j \in \mathcal{J}, \forall k \in \mathcal{K}$ :

$$
\sum_{\tilde{k}=1}^{K} d_{j, \tilde{k}}+\sum_{\tilde{j}=1}^{J} d_{\tilde{j}, k}-d_{j, k} \leq 1 .
$$

This result can be written in an alternative form as

$$
\sum_{\tilde{k}=1}^{K} d_{j, \tilde{k}}+\max _{k} \sum_{\tilde{j} \in \mathcal{J}, \tilde{j} \neq j}^{J} d_{\tilde{j}, k} \leq 1, \quad \forall j \in \mathcal{J} .
$$

1) $K \times 2 X$ channel

Comparing (7) and (28), it can be seen that the inner bound does not meet the outer bound in general. However, there are some special cases where they do meet. One such case is when $J=2$. In this case, both bounds are given by

$$
\begin{aligned}
& \sum_{\tilde{k}=1}^{K} d_{1, \tilde{k}}+\max _{k} d_{2, k} \leq 1, \\
& \sum_{\tilde{k}=1}^{K} d_{2, \tilde{k}}+\max _{k} d_{1, k} \leq 1 .
\end{aligned}
$$

We summarize the result in the following.

Theorem 3 (DoF Region of $K \times 2 \mathrm{X}$ Network). The DoF region of the $K \times J$ wireless $X$ network when $J=2$ is a.e. the set of $\left[d_{j, k}\right]_{j=1, k=1}^{2, K}$ that have non-negative entries and satisfy both (29) and (30).

\section{2) Some boundary points on the general DoF region}

Another case where the two bounds (7) and (28) meet is when $d_{j, k}=d_{j, \hat{k}}$, for all $j \in \mathcal{J}$ and for all $k, \hat{k} \in \mathcal{K}$. We have:

Theorem 4 (Some Boundary Points). The DoF region of the $K \times J$ wireless $X$ network a.e. has the following points on the boundary: $\left[d_{j, k}\right]_{j=1, k=1}^{J, K}$ such that

i) all entries are non-negative;

ii) $d_{j, k}=d_{j, \hat{k}}$, for all $j \in \mathcal{J}$ and for all $k, \hat{k} \in \mathcal{K}$;

iii) $(K-1) \max _{j \in \mathcal{J}} d_{j, 1}+\sum_{j \in \mathcal{J}} d_{j, 1}=1$.

This is true for Lebesgue almost everywhere $\mathbf{H} \in \mathbb{R}^{J \times K}$.

If we set all $d_{j, k}=1 /(J+K-1)$, then we recover the total DoF of $d^{\text {(total) }}=J K /(J+K-1)$ of [1], [9].

\section{B. Extensions}

It is possible to extend the result in the paper to cases where each transmitter emits an arbitrary number of messages, and each receiver may request an arbitrary subset of the messages emitted by all the transmitted. This can be termed the wireless $\mathrm{X}$ network with multicast, or wireless $\mathrm{X}$ network with general message demands. For the case where each transmitter emits only one message, and the channel varies with time, the DoF region for time-varying channel has been obtained in [11]. The same DoF region, but for a constant coefficient channel, can be derived using the technique developed in this paper. As a special case of that, the DoF region result of $K$-user interference channel with single antennas [12] can also be recovered.

\section{CONCLUSIONS}

We have derived some achievability results for the wireless $\mathrm{X}$ network with single antennas. Each message is split into multiple streams, and achievability is established using real interference alignment of the streams. The streams emitted by a single transmitter can be "shuffled" to determine the alignment position with respect to streams from other transmitters. Such rearrangement allow for higher DoF in some cases. It is not known whether the presented schemes are sufficient to achieve all points in the DoF region. However, we showed that when the number of receivers is equal to two, then the achieved region is actually the DoF region. We also showed that certain boundary points in the general DoF region can be achieved using the proposed schemes. Closing the possible gap would be a meaningful objective. It would also be interesting to investigate the multiple antenna cases. Quantifying the DoF region of the general wireless $\mathrm{X}$ network with arbitrary number antennas at each node, and with general message demands, would be a useful result.

Acknowledgement: The work in this paper was supported in part by the NSF grant No. 1128477.

\section{REFERENCES}

[1] V. R. Cadambe and S. A. Jafar, "Interference alignment and the degrees of freedom of wireless x networks," IEEE Trans. Inform. Theory, vol. 55, no. 9, pp. 3893-3908, Sept. 2009.

[2] S. A. Jafar and S. Shamai, "Degrees of freedom region of the MIMO x channel," IEEE Trans. Inform. Theory, vol. 54, no. 1, pp. 151-170, Jan. 2008.

[3] L. Zheng and D. Tse, "Communicating on the Grassmann manifold: A geometric approach to the non-coherent multiple antenna channel," IEEE Trans. Inform. Theory, vol. 48, no. 2, pp. 359-383, Feb. 2002.

[4] M. A. Maddah-Ali, A. S. Motahari, and A. K. Khandani, "Signaling over MIMO multi-base systems: Combination of multi-access and broadcast schemes," in Proc. IEEE Intl. Symp. on Info. Theory, pp. 2104-2108, 2006.

[5] V. R. Cadambe and S. A. Jafar, "Interference alignment and degrees of freedom of the k-user interference channel," IEEE Trans. Inform. Theory, vol. 54, no. 8, pp. 3425-3441, Aug. 2008.

[6] G. Bresler, A. Parekh, and D. N. C. Tse, "The approximate capacity of the many-to-one and one-to-many gaussian interference channels," IEEE Trans. Inform. Theory, vol. 56, no. 9, pp. 4566-4592, Sept. 2010.

[7] S. Sridharan, A. Jafarian, S. Vishwanath, S. A. Jafar, and S. Shamai, "A layered lattice coding scheme for a class of three user gaussian interference channels," in 46th Annual Allerton Conference Control, and Computing, on Communication, pp. 531-538, 2008.

[8] R. H. Etkin and E. Ordentlich, "On the degrees-of-freedom of the kuser gaussian interference channel," CoRR, vol. abs/0901.1695, 2009; [online http://arxiv.org/abs/0901.1695]

[9] A. S. Motahari, S. O. Gharan, M. A. Maddah-Ali, and A. K. Khandani, "Real interference alignment: Exploiting the potential of single antenna systems," CoRR, vol. abs/0908.2282, 2009; [online http: / /arxiv.org/abs/0908.2282]

[10] H. Yin, L. Ke, and Z. Wang, "Interference alignment and degrees of freedom region of cellular sigma channel," in Proc. IEEE Intl. Symp. on Info. Theory, Saint Petersburg, Russia, July 2011 (accepted); [online http: / /arxiv.org/abs/1103.0270]

[11] L. Ke, A. Ramamoorthy, Z. Wang, and H. Yin, "Degrees of freedom region for an interference network with general message demands," IEEE Trans. Inform. Theory, Jan. 2011 (submitted); [online http: / /arxiv.org/abs/1101.3068]

[12] Y. Wu, S. Shamai(Shitz), and S. Verdú, "Degrees of freedom of interference channel: a general formula," in Proc. IEEE Intl. Symp. on Info. Theory, 2011. 